\title{
Cytokine-treated Veto Cells
}

National Cancer Institute

\section{Source}

National Cancer Institute. Cytokine-treated Veto Cells. NCI Thesaurus. Code C153337.

A preparation of activated veto cells, with potential immunostimulating and antineoplastic activities. White blood cells (WBCs), taken from either the patient or a healthy third-party donor, are processed and ex-vivo treated with an as of yet not disclosed mix of cytokines to activate specific cytotoxic veto cells. Upon administration, the veto cells specifically target and bind to tumor or foreign cells, thereby inducing apoptosis. Veto cells are able to selectively activate the immune system to only attack and kill tumor or foreign cells upon transplantation to prevent graft-versus-host disease (GvHD). 